\title{
BCG Therapy in Non-Infiltrating Bladder Tumor: About 63 Cases, Experience of Urology «A » Department
}

Abdallah Hussein*, Dergamoun Hamza, El Boukili El Makhoukhi Zayd, Hachem Al Sayegh, Benslimane Lounis, Nouini Yassine

Department of Urology A, Ibn Sina University Hospital Center, Morocco

DOI: $10.36347 /$ sasjs.2020.v06i04.006

| Received: 23.03.2020 | Accepted: 03.04.2020 | Published: 11.04.2020

*Corresponding author: Abdallah Hussein

Abstract

Bladder carcinoma is diagnosed or treated worldwide in 2.7 million people each year and, in the majority of cases, urothelial tumors appear after 60 years. Over a period of 4 years from 2016 to 2019, 63 patients benefited from BCUV adjuvant therapy at RTUV. The average age of the patients is 58 years. The sex ratio is 5.7 predominantly male Smoking was found in $66 \%$ of patients. The predominant reason for consultation is hematuria in $87.2 \%$. Ultrasound of the urinary tract showed a bladder bladder tumor in 49 patients, with bladder thickening in 10 patients and bladder bladder disease in 4 patients. During cystoscopy with diagnostic RTUV, the tumor was unifocal in $38.2 \%$ and multifocal in $61.7 \%$. In the pathology study, it was urothelial carcinoma in 62 patients, only 1 patient had carcinoma in situ and 6 patients had the same lesions at the same time. All patients benefited from a complete RTUV followed by BCG therapy. The most used product is BCG culture at a dose of $120 \mathrm{mg}$ per instillation. The protocol used is one instillation / week for 6 weeks followed by another series of 3 weekly instillations after a rest of 6 weeks, then maintenance schedule on the basis of 3 weekly instillations at 3 months, 6 months, then every 6 months for 3 years. 4 patients experienced side effects from BCG therapy. RTUV with surveillance was performed on his patients. Cystoscopy and urine cytology are the monitoring methods used. The control cystoscopy showed a recurrence in 10 patients. Progression of the tumor was found in 7 patients. The therapeutic alternative is discussed case by case.

Keywords: Bladder carcinoma, urothelial tumors, RTUV, ystoscopy.

Copyright @ 2020: This is an open-access article distributed under the terms of the Creative Commons Attribution license which permits unrestricted use, distribution, and reproduction in any medium for non-commercial use (NonCommercial, or CC-BY-NC) provided the original author and source are credited.

\section{INTRODUCTION}

Urothelial tumors are the most common tumors of bladder tumors. It is $90 \%$ of cases of urothelial carcinoma or transitional cell carcinoma. They constitute the 11 th cause of cancer in the world and the 2 nd urological cancer after prostate cancer.

Non-muscle infiltrating bladder tumors (NMIBT) make up $70 \%$ of bladder cancers. Their penetration does not exceed the chorion of the mucosa but can however become invasive. A distinction is made between plane lesions (CIS); tumors limited to the mucosa (Ta) and tumors reaching the chorion without exceeding the lamina propria.

Tobacco and certain industrial agents are the main factors responsible for their induction.

Cystoscopy and anatomopathological analysis of the resection cups are the key diagnostic examinations.
The main risks of these tumors after resection are recurrence in the same superficial mode or progression becoming infiltrating much more aggressive than the initial tumor.

Their treatment is essentially based on transurethral resection of the bladder (RTUV) associated with endovesical instillations by BCG (Bacillus Calmette-Guerin) therapy or local chemotherapy which is necessary in order to reduce these risks.

BCG endovesical therapy is the most commonly used treatment, it significantly reduces the risk of progression and recurrence. It can generate some number of complications, some of which are serious, hence the importance of respecting its indications and instructions for use and of carefully monitoring its tolerance and efficacy. 


\section{MATERIALS AND METHODS}

The study took place in the urology department of Ibn Sina Rabat hospital. This is a prospective, non-randomized, single-center study of 63 patients over a period of 4 years, from 2016 to 2019 .

All patients with non-muscle infiltrating bladder tumor who received BCG therapy were included in our study

We made a farm report on which all the data collected were reported. This data comes from patient registers, medical records during hospitalization. Data regarding the feasibility of BCG therapy were collected from a doctor on the ward.

\section{THE OPERATING SHEET}

Identity:

Last name and first name:

Gender: $\mathrm{M} \square \mathrm{F} \square$

Age: .......... years

Risk factors: Smoking $\square$ Industry $\square$ No FDR $\square$

The primary tumor:

Macroscopic study of the tumor:

Number: no tumor $\square$ single tumor $\square$ multiple tumor $\square$

Seat: retrotrigonal $\square$ lateral $\square$ trigonal $\square$

dome $\square$ multifocal tumor $\square$

carcinoma in situ: associated $\square$ no $\square$

Synchronous or metachronous TVE: yes $\square$ no $\square$

Size: $<3 \mathrm{~cm} \square$ more than $3 \mathrm{~cm} \square$

Pathology:

Histological type: Tm uroth $\square$ Tm non uroth $\square$
Stage: Ta $\square \mathrm{T} 1 \square \mathrm{T} 2 \square$ Muscle not seen $\square$

Grade: low grade $\square$ high grade $\square$

Time to first recurrence / progression:

Treatment:

RTUV only: yes $\square$ no $\square$

BCG induction treatment only: yes $\square$ no $\square$

BCG induction treatment + maintenance: yes $\square$ no $\square$

The secondary tumor:

Number: no tumor $\square$ single tumor $\square$ multiple tumor $\square$ Size: ..in $\mathrm{cm}$.

The new stage: Ta $\square \mathrm{T} 1 \square \mathrm{T} 2 \square$ Muscle not seen Ta $\square$ The new grade: low grade $\square$ high grade $\square$

Lymph node invasion: yes $\square$ no $\square$

Treatment of secondary tumor:

A new instillation of BCG therapy: yes $\square$ no $\square$

Cystectomy: yes $\square$ no $\square$

\section{RESULTS}

Demographic Data

Age

The average age of the patients was 58 years.

Age distribution:

$\varnothing$ From 40 years to 50 years: 10 cases / $63.15 .87 \%$

$\emptyset$ From 51 years to 60 years: 33 cases / 63. $52.38 \%$

$\varnothing$ From 61 years to 70 years: 14 cases / 63. $22.22 \%$

$\varnothing$ From 71 years to 80 years: 6 cases / 63. 9.52\%

Sex

The sex ratio is 6 , predominantly male.

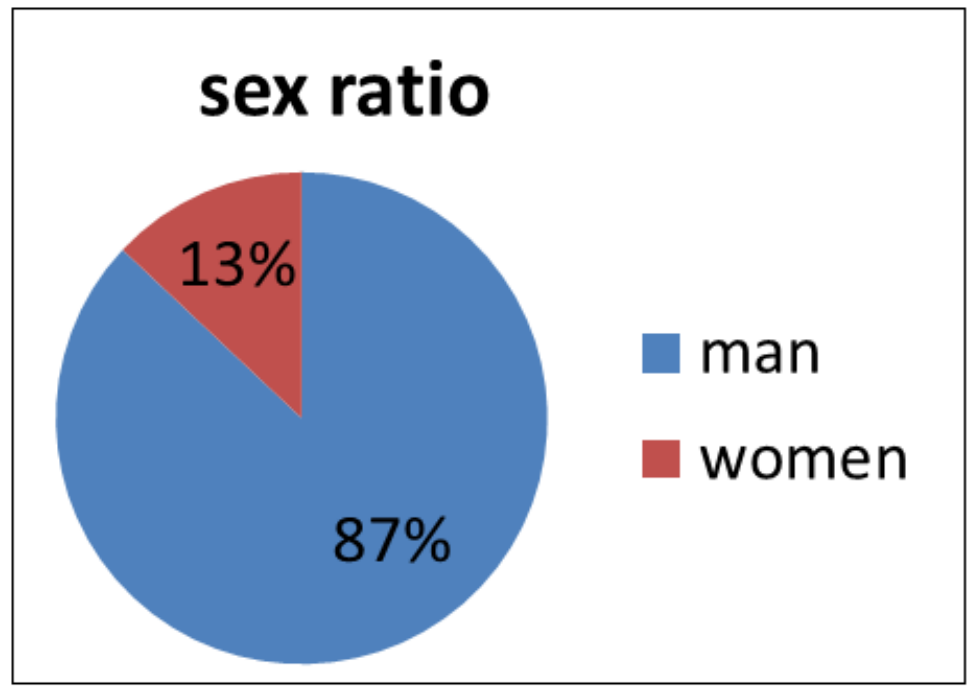

Fig-1: Distribution of the sexes in our series

\section{CLINICAL DATA}

\section{Distribution according to WHO risk factors}

- Smoking over 10 packs / year:

- Industry (rubber, coal, paint, etc.)

- Lack of risk factors. 


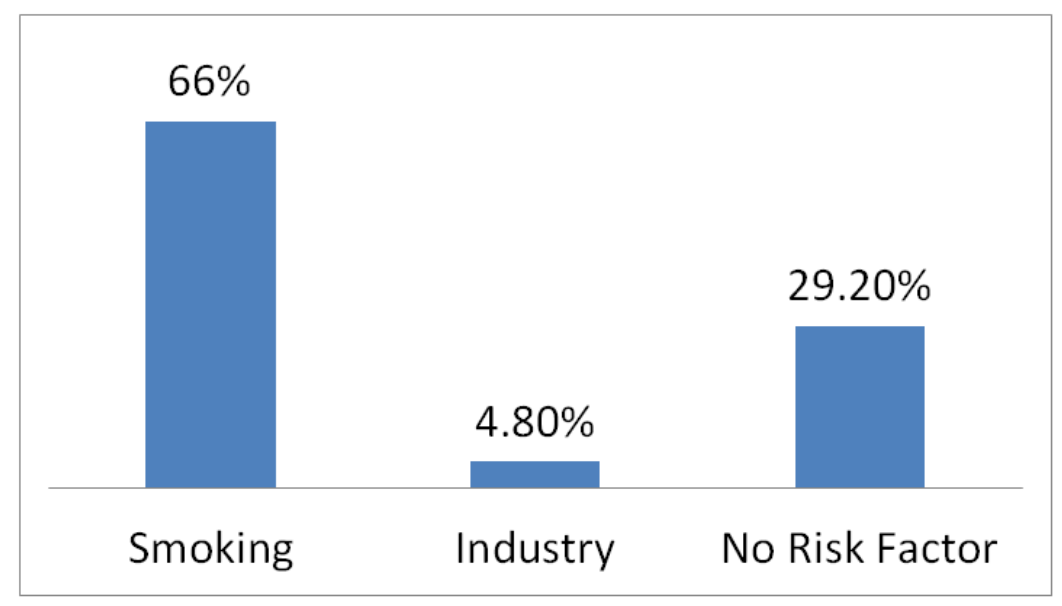

Fig-2: Distribution of patients according to WHO risk factors

\section{Diagnostic Circumstances}

The predominant reason for consultation was gross hematuria in $87.2 \%$, followed by bladder filling disorder (TRV) in $42.5 \%$ and then acute urine retention (RAU) in $4.25 \%$. The association of several clinical signs was found in $55.3 \%$.

\section{Paraclinical Data \\ Radiological Assessment}

In our series, all patients underwent imaging of the bladder and excretory urinary tract, of which 53 patients underwent a bladder and excretory ultrasound and 10 patients underwent a url-scanner.

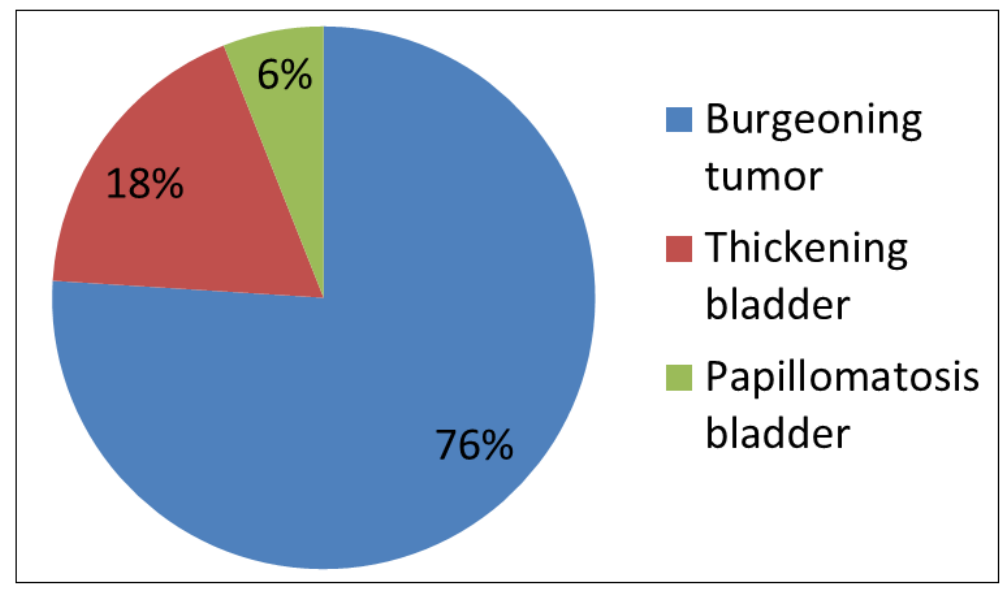

Fig-3: Appearance of the bladder tumor

\section{Endoscopic Assessment: Diagnostic Cystoscopy}

A single tumor location was mentioned in 18 patients (20\%), and multifocal in 44 patients $(80 \%)$.

The lateral wall is the most frequent localization in our series $(36.1 \%)$, followed by the bladder fund (23.4\%), bladder trigone (12.7\%), the bladder neck $(8.5 \%)$, perieatic location in 3 patients $(6.3 \%)$ and bladder papillomatosis in 4 patients $(8.5 \%)$.

In our series, the papillary aspect was the most frequent $(87.2 \%)$ of which 4 patients had a tumor appearance in cauliflower designating papillomatosis vesicale.

The macroscopic appearance of carcinoma in situ (CIS) has only been described in 6 patients (10\%).
20 patients had less than three tumor locations and 24 patients had more than three locations.

\section{RTUV and Anatomopathology}

The anatomopathological examination of the transurethral resection coupons (RTUV) was carried out in order to carry a complete identification of the tumor: its histological type, its stage and its grade.

\section{Histological Type}

In our series, the anatomopathological study after transurethral resection revealed only one histological type: urothelial carcinoma, which was papillary in all patients except one patient who had carcinoma in situ (CIS). 


\section{Histological Grade}

The histological grade was classified according to the latest WHO classification in 2004 as a low grade tumor and a high grade tumor.

In our series, $30 \%$ of the patients had a low grade tumor and $70 \%$ had a high grade tumor.

\section{Histological Stage}

According to the WHO TNM classification of bladder tumors, non-muscle infiltrating tumors (TVNIM) correspond to stage Ta, Tis and T1.

In our series, the majority of patients had a T1 tumor $(63 \%)$, followed by Ta tumors $(35 \%)$ and only one patient had a Tis.

\section{Risk of recurrence and progression}

Using the 6 main clinicopathological parameters (cell grade, tumor stage, tumor size, delay in tumor recurrence, concomitant CIS, number of tumors and multifocality), it is possible to estimate the risk of tumor recurrence and muscle progression of TVNIM. In our series, the majority of patients had a high or intermediate risk.

\section{The protocol of endovesical instillations} The delay of instillations after RTUV:

It varies between 4 and 6 weeks, the majority of patients have achieved the first instillation of BCG therapy 6 weeks after a complete RTUV.

\section{Product and dose used:}

The most used product in our service is BCG culture ${ }^{\circledR} 120 \mathrm{mg}$. ImmuCyst ${ }^{\circ} 81 \mathrm{mg}$ is out of the market which has reduced its use.

Another recent product that has become increasingly popular is OncoBCG SII ${ }^{\circledR} 120 \mathrm{mg}$, which is an Indian strain of BCG.

The recommended dose is $120 \mathrm{mg} /$ instillation and this is the dose used for all of our patients.

\section{Cystoscopy and pre-therapeutic RTUV:}

In our series, all the patients benefited from a pre-therapeutic cystoscopy the aim of which is to eliminate all tumor residue and also to make a complete RTUV before starting the endovesical instillations.

\section{Insulation Protocols \\ Induction Diagram}

The induction scheme carried out in our service contains 6 weekly instillations at the start of induction and 3 weekly instillations at the end of induction after a period of 6 weeks.

More than half of the patients (55.3\%) completed the 6 recommended instillations at the start of induction. The end-of-induction regimen was performed in $42.5 \%$ of patients.

\section{Maintenance Schedule}

- The maintenance schedule contains 1 to 3 weekly instillations at 3 months, 6 months, 12 months, 18 months, 24 months.

- In our series, 14 patients had maintenance instillations.

\section{Side Effects}

The side effects of instillations are sought after each instillation during the following consultation.

In our series, we collected 10 patients who had side effects from BCG therapy. 3 patients with hematuria and 7 have urinary problems.

For hematuria we decided to stop the instillation is to redo a cystoscopy and the other we treated symptomatically or the symptom disappeared in 5 days.

\section{Evolution}

\section{Control Cystoscopy}

Cystoscopy is a fundamental tool for monitoring bladder tumors, in particular for TVNIM, it constitutes a means of prognostic evaluation and therapeutic response under BCG therapy.

In our series, 25 patients $(40 \%)$ had a normal cystoscopy during the study period, a recurrence was mentioned in 15 cases (24\%), of which 5 were Ta stage and 10 were stage $\mathrm{T} 1$; and an increase was found in 11 cases $(17 \%)$.

The outcome of the control cystoscopy was not reported in 12 patients.

\section{Control cytology}

Monitoring by urinary cytology made that In Eight Patients in Our study for an economic reason. Which came out positive in two patients.

\section{Other Therapies Envisaged}

Adjuvant therapy with BCG therapy had to be discontinued in 22 patients for the following reasons:

- The finding of a tumor at high risk of recurrence and progression: 11 cases

- The tumor has become infiltrating: 3 cases

- Lack of means to obtain treatment: 5 cases

- $\quad$ Side effects of BCG therapy: 3 cases

The therapeutic alternatives depend on the reason why BCG therapy was abandoned.

In our series, we collected 14 patients whose therapeutic alternative was to redo RTUV 
Three patients underwent a total cystoprostatectomy with adjuvant radio-chemotherapy in front of the progression of the tumor which became infiltrative.

Abstention and surveillance was the therapeutic alternative for 5 patients who could not afford the treatment.

\section{DISCUSSION}

The choice of immunotherapy in the face of an evolving risk TVNIM implies rigorous management [1].

- Patient selection

- The realization of the instillations

- Analysis of the results of the initial treatment

Endovesical immunotherapy is an adjunctive therapy to endoscopic resection (RTUV) of a nonmuscle infiltrating bladder tumor (TVNIM).

These indications concern all major risk tumors and certain intermediate risk tumors, namely [2]:

- TaG2G3 multi-recurrent tumors;

- T1 tumors;

- The CIS

Indeed, it is used for a purpose:

- Prophylactic: urothelial carcinomas Ta, T1;

- Therapeutic: carcinoma in situ.

Currently, BCG is both a preventive treatment for recurrence and progression of high-risk TVNIM, and a highly effective curative treatment for carcinoma in situ [2].

The occurrence of post-therapeutic
$\begin{aligned} & \text { complications is linked to the various BCG } \\ & \text { preparations. }\end{aligned}$

LAMM had demonstrated, after a study involving 2602 patients, the main side effects depending on the type of BCG therapy used [3]. According to LAMM, there is no statistically significant difference between the different strains of BCG concerning the occurrence of local side effects. Locoregional and general complications do not appear to be more frequent, regardless of the strain used.

A study was carried out by CHAMPERTIER on a series of 89 patients in order to compare the toxicity of the new strain (Connaught) with that previously used in France (Pasteur strain).

This study had not shown a significant difference in toxicity between the two strains, on the other hand another study made by COLOMB had concluded that the Immunocyst seems better tolerated than the BCG Immun Pasteur.

In addition, the study by S. Holz showed that the number of undesirable effects and severe undesirable effects was reduced significantly over time, potentially following the change from the Connaught strain (Immucyst®) to the Tice strain (Oncotice $\left.{ }^{\circledR}\right)$ [4].

The study of the side effects of BCG according to the start date of the instillations shows a significantly higher rate of local BCGite in the case of early treatment, with a percentage of systemic manifestations comparable in the two groups [5].

The side effects of BCG therapy are classified into:

- Minors: finding during less than 48 hours of pollakiuria, urination, urinary burns, gross hematuria, fever below $38^{\circ} \mathrm{C}$.

- Means: symptomatology immediately severe or lasting more than 48 hours. In this case it is necessary to suspend any instillation until complete resolution of the symptoms, then resumed with reduction of the doses to $1 / 3$ or even $1 / 10$ of the initial dose.

- Major: fever $>38^{\circ}$ 5, intense and prolonged bladder irritant symptoms, prostatitis, epididymitis, visceral tuberculosis and generalized BCGitis. These complications necessitate the permanent cessation of treatment [6].

Instill therapy with BCG should be considered a failure in the following clinical situations:

- When a tumor infiltrating the muscle is detected during systematic monitoring.

- If a high-grade tumor that does not infiltrate the muscle is present at 3 months and 6 months.

- Any aggravation of the bladder disease under treatment with BCG: a multifocal recurrence, a tumor stage or a higher cell grade; the persistence of carcinoma in situ despite an initial response.

The patient contributes significantly to the success of the treatment, he must be informed about the interest of BCG therapy, the protocol of its administration, its cost and possible side effects.

Currently, BCG induction therapy is started at least four weeks after the last tumor resection.

In our department, once the indication for a treatment by endovesical instillation of BCG therapy is posed, the instillations are started 4 to 6 weeks after a complete RTUV and in the absence of any gross hematuria and any urinary tract infection. 
The purpose of the pre-therapeutic assessment is to eliminate all contraindications to the achievement of endo-vesical instillation of BCG therapy. It is first clinical, asking the patient about the presence of hematuria, signs of bladder irritation, a history of a systemic reaction to BCG therapy and traumatic catheterization. Then, the paraclinical pre-therapeutic assessment, in particular a cystoscopy which aims to search for a tumor residue, a bladder perforation, and also to carry out a RTUV in the event of a residue.

ECBU is also essential before each instillation in order to eliminate a urinary tract infection which will require antibiotic treatment and a control ECBU before starting BCG therapy.

BCG culture SSI ${ }^{\circledR}$ is the most widely used product in the urology department at the Ibn Sina Rabat University Hospital.

Endovesical instillations of BCG therapy can be performed by a qualified urologist or by a urology resident. The instillation takes place on an outpatient basis in a specific room.

After 4-6 weeks of resection, induction therapy with BCG is started. It is six weekly instillations of 81 mg of Immucyst® (start of induction), supplemented by three weekly instillations of $120 \mathrm{mg}$ of BCG SII after six weeks of discontinuation (end of induction) [3, 7, 8].

BCG maintenance therapy appears to be the option of choice to decrease both the risk of recurrence and tumor progression. The modalities of this treatment were not clearly defined. Various protocols have been tested. The only available randomized study showing an advantage of maintenance treatment in terms of recurrence-free survival and tumor progression was carried out by Lamm et al. [9] from SWOG (South West Oncology Group). The protocol used consisted of 3 weekly instillations at 3, 6, 12, 18, 24, 30 and 36 months.

This last protocol is the one adopted in our practice. However, it has been noted that only one patient completed their BCG therapy sessions at 12 months. This can be explained by the reduced duration of the study and by the delay in instillations for socioeconomic and therapeutic reasons (availability of treatment, side effects, tumor progression or recurrence).

Given the risks attributable to treatment, a specific assessment at the start of treatment is recommended and each new instillation is preceded by a clinical examination, an assessment of the tolerance of previous instillations and an ECBU (<4 days) [10].
Regarding oncology follow-up, cystoscopy coupled with cytology, remains the benchmark examination for surveillance of TVNIM.

In addition, other diagnostic tools could help alleviate this endoscopic monitoring. Regarding tumor markers, numerous urine tests are available [11]. Among the most effective urine tests, we can cite: BTA ${ }^{\circ}$ (bladder tumor antigen), NMP22®, ImmunoCyt ${ }^{\circledR} \quad$ combining cytology and immunofluorescence, UroVysion ${ }^{\circledR} \mathrm{m}$. These tests require a specialized technical platform and a deeper knowledge of their indications which constitutes a limit to their practical use.

In our daily practice, urine cytology and cystoscopy keep their place in the follow-up of the TVNIM because of their satisfactory cost / effectiveness ratio.

The pace and the means used for surveillance of TVNIM have been adapted to their risk of recurrence and progress established according to the tables of the EORTC. The rhythm of endoscopic surveillance of low risk TVNIM (EORTC <7) is justified by the rate of recurrence $(23.7 \%$ for pTa-1 lesions with low potential for malignancy or low grade), of aggravation (4, 7\% of tumors progressing towards the high grade) and progression $(2.6 \%$ of tumor progressing towards a TVIM) [12]. For this subgroup, only a cystoscopic examination is justified at 3 and 12 months then annually for 10 years (for life in the event of continued smoking intoxication). The use of fluorescence cystoscopy is not warranted for this low-risk subgroup. Conversely, surveillance for high-risk lesions (EORTC> 14) must be carried out more closely, using at the same time fibroscopy, imaging and cytology. This attitude is justified by the risk of recurrence and progression at 1 year high (from 30 to $61 \%$ and 11 to $17 \%$ respectively). Monitoring should be extended due to a long-term risk of recurrence ( 49 to $78 \%$ at 5 years) and progression (26 to $45 \%$ at 5 years) $[13,14]$. Cystoscopy and cytology must therefore be performed quarterly in the first year, then semi-annually in the second year, then annually for life. Uro-CT will be performed every 2 years or in case of positive cytology or symptom suggestive of TVES. Monitoring of intermediate risk lesions (EORTC 7-13) is also adapted to the risk of recurrence and progression.

The cystoscopic evaluation of patients in our series is also done according to the risk of recurrence and progression but also adapt to the rhythm of the realization of BCG therapy.

The risk of recurrence and progression depends on several parameters, in particular the stage, grade, multifocality and the average time for follow-up of patients, which explains the variability of the percentages found in the different studies. 
Regarding Ta tumors, Martinache objectified in a retrospective study of 193 patients that pTa bladder tumors are associated with a high risk of recurrence. After an average follow-up of 58 months, 56.5\% of his patients presented at least one recurrence and $9.3 \%$ presented progression of the lesions. Which was close to the results reported by other authors [15].

In our study, among the Ta tumors, we collected only one case of recurrence and 2 cases of progression, this difference can be explained by the reduced number of patients and the short duration of follow-up.

For T1 tumors, BCG therapy occupies a prominent place. Several studies have affirmed the role of immunotherapy in reducing the rate of recurrence and tumor progression. However, there is a great disparity in the rates displayed [16].

These differences are explained for several reasons $[17$, 18]:

- The patients were not all selected in the same way, in particular for the oldest studies where the second resection was not systematic.

- Follow-up periods range from 23 months to 15 years. It is likely that for studies with limited follow-up, a longer duration of observation will see the rates of recurrence and / or progression increase.

- The instillation protocols vary from one study to another and within the same series.

The rates of complications linked to endovesical instillations of BCG reported in the literature are very variable.

In our series, we only collected three patients who had minor side effects to BCG therapy. These complications occurred during the first cycle of BCG therapy. Which justified the abandonment of this treatment.

Our results are different from those reported by HOLZ et al., who objectified in his study of 146 patients, that $20.6 \%$ of patients stopped treatment due to side effects. It was also observed that in $80 \%$ of the cases, the undesirable effects which led to the discontinuation of the treatment occurred during the first 3 cycles of BCG [4].

Carrying out BCG therapy may be subject to constraints that are responsible for stopping treatment. Its constraints are linked either:

- Tumor: tumor that has become infiltrating or at high risk of recurrence and progression.

- To the patient: non-adherence to treatment, intolerance of treatment, socio-economic level

- To the product: its cost and its unavailability
The therapeutic alternatives depend on the above-mentioned reasons. For patients intolerant to $\mathrm{BCG}$, it is recommended to reduce the doses for low to intermediate risk tumors and to maintain a standard dose for high risk tumors associated with prophylaxis with Ofloxacin 6 hours after instillation of BCG [19].

In our series, in the 3 patients who presented side effects, the attitude was to stop BCG therapy and to do a cystoscopic monitoring with RTUV.

For the 11 patients whose tumor became at high risk of recurrence and progression, the attitude was to repeat an RTUV and to restart the BCG therapy protocol, but in the 3 patients whose tumor progressed and became infiltrative, one Total cystoprostatectomy was performed followed by radio-chemotherapy.

\section{CONCLUSION}

Urothelial tumors are one of the common tumors; At the initial diagnosis, $70 \%$ of the tumors are TVNIM, $25 \%$ are invasive and $5 \%$ are metastatic. Among the superficial tumors, 60 to $70 \%$ will recur and 10 to $20 \%$ will progress to tumors which invade the muscle becoming potentially metastatic. This unfavorable development of initially superficial tumors must be detected as soon as possible. Upstream, prevention through the fight against smoking and occupational risk factors is essential.

Advances in the management of TVNIM in recent years have improved outcomes in terms of recidivism and progression. Among the accepted rules, we find the systematic realization of an IPOP in TVNIM (except against indication), the second resection for high risk TVNIM, the growing interest of the resection coupled with fluorescence. BCG therapy is currently considered to be the adjuvant therapy of choice after total RTUV in the treatment of TVNIM. Several studies have shown its superiority compared to other treatments. Colossal progress has been made in the optimal scheme of endovesical instillations at BCG. The main objective was to maintain a fair and satisfactory balance between efficacy and tolerance because it can also cause side effects which are rare but sometimes serious, hence the advantage of detecting them early and knowing how to treat them and above all warn them.

However, BCG therapy is subject to constraints related to both the patient and the treatment itself. This can lead to an abundance of treatment and the switch to another therapeutic alternative.

\section{REFERENCES}

1. Brosman SA. Bacillus Calmette-Guerin immunotherapy. Techniques and results. The Urologic clinics of North America. 1992 Aug;19(3):557-64. 
2. Chopin D. Traitement des tumeurs superficielles de la vessie: Cancers des voies excrétrices urinaires. La Revue du praticien (Paris). 1997;47(4):382-7.

3. Argyropoulos AN, Tolley DA. Upper urinary tract transitional cell carcinoma: current treatment overview of minimally invasive approaches. BJU international. 2007 May;99(5):982-7.

4. Holz S, Sotorres JC, Legrand F, Gilsoul J, Pirson $\mathrm{M}$, Roumeguère $\mathrm{T}$. Evaluation of adverse events caused by intravesical BCG instillations: Has the strain used a potential implication?. Progres en urologie: journal de l'Association francaise d'urologie et de la Societe francaise d'urologie. 2016 Feb;26(2):73-8.

5. Neuzillet $Y$, Roupret M, Wallerand H, Pignot G, Larré S, Irani J, Davin JL, Moreau JL, Soulié M, Pfister C. Diagnostic et prise en charge des événements indésirables survenant au décours des instillations endovésicales de BCG pour le traitement des tumeurs de vessie n'infiltrant pas le muscle (TVNIM): revue du comité de cancérologie de l'Association française d'urologie. Progrès en urologie. 2012 Dec 1;22(16):989-98.

6. Ricshmann P, Mazerottes C, Bouchot O, Chopin DK. Carcinomes urothéliales de vessie T1G3: Résultats du traitement d'induction par BCG. Etude multicentrique prospective. Prog Urol, 1999;9(1):5.

7. Allouc H, Benoît G, Paradis V, Blanchet P, Jardin A. Le risque de complications au cours du traitement endovésical par le BCG pour tumeur superficielle de vessie. La Presse médicale (1983). 1997;26(27):1284-8.

8. BILLERY C. Classification histologique et marqueurs pronostiques : tumeurs de vessie. Prog urol 1998.

9. Lamm DL, Blumenstein BA, Crissman JD, Montie JE, Gottesman JE, Lowe BA, Sarosdy MF, Bohl RD, Grossman HB, Beck TM, Leimert JT. Maintenance bacillus Calmette-Guerin immunotherapy for recurrent TA, T1 and carcinoma in situ transitional cell carcinoma of the bladder: a randomized Southwest Oncology Group Study. The Journal of urology. 2000 Apr;163(4):1124-9.

10. Colin P, Neuzillet Y, Pignot G, Roupret M, Comperat E, Larre S, Roy C, Quintens H, Houedé N, Soulié M, Pfister C. Surveillance des carcinomes urothéliaux: revue du Comité de cancérologie de l'Association française d'urologie. Progrès en Urologie. 2015 Sep 1;25(10):616-24.

11. Ayed M, Ben Hassine L, Ben Slama R, CHELBI N, GHOZZI S. Résultats du BCG dans le traitement des tumeurs de vessie pTa et pT1. Evaluation d'un protocole long utilisant $75 \mathrm{mg}$ de BCG de souche Pasteur. Progrès en urologie (Paris). 1998;8(2):206-10.

12. Linton KD, Rosario DJ, Thomas F, Rubin N, Goepel JR, Abbod MF, Catto JW. Disease specific mortality in patients with low risk bladder cancer and the impact of cystoscopic surveillance. The Journal of urology. 2013 Mar 1;189(3):828-33.

13. Sylvester RJ, van der Meijden AP, Oosterlinck W, Witjes JA, Bouffioux C, Denis L, Newling DW, Kurth K. Predicting recurrence and progression in individual patients with stage $\mathrm{Ta} \mathrm{T} 1$ bladder cancer using EORTC risk tables: a combined analysis of 2596 patients from seven EORTC trials. European urology. 2006 Mar 1;49(3):46677.

14. Fernandez-Gomez J, Madero R, Solsona E, Unda M, Martinez-Piñeiro L, Ojea A, Portillo J, Montesinos M, Gonzalez M, Pertusa C, Rodriguez-Molina J. The EORTC tables overestimate the risk of recurrence and progression in patients with non-muscle-invasive bladder cancer treated with bacillus CalmetteGuérin: external validation of the EORTC risk tables. European urology. 2011 Sep 1;60(3):42330 .

15. Martinache G, Zerbib M, Descazeaud A, Debré B, Peyromaure M. Tumeurs de vessie pTa: facteurs de récidive et de progression. Progrès en urologie. 2008 Jan 1;18(1):35-40.

16. Van Rhijn BW, Burger M, Lotan Y, Solsona E, Stief CG, Sylvester RJ, Witjes JA, Zlotta AR. Recurrence and progression of disease in nonmuscle-invasive bladder cancer: from epidemiology to treatment strategy. European urology. 2009 Sep 1;56(3):430-42.

17. Shahin O, Thalmann GN, Rentsch C, Mazzucchelli L, Studer UE. A retrospective analysis of 153 patients treated with or without intravesical bacillus Calmette-Guerin for primary stage T1 grade 3 bladder cancer: recurrence, progression and survival. Journal Urol, 2003;169:96-100.

18. Patard JJ, Rodriguez A, Leray E, Rioux-Leclercq $\mathrm{N}$, Guille F, Lobel B. Intravesical Bacillus Calmette-Guerin treatment improves patient survival in T1G3 bladder tumours. Eur Urol, 2002;41:635-42.

19. Pfister C, Roupret M, Wallerand H, Davin JL, Quintens H, Guy L. Recommandations du CCAFU 2010: Tumeurs urothéliales. Prog Urol. 2010;20:S255-74. 